\title{
Pulsed radiofrequency treatment of the lumbar dorsal root ganglion in patients with chronic lumbar radicular pain: a randomized, placebo-controlled pilot study
}

\author{
This article was published in the following Dove Press journal: \\ Journal of Pain Research \\ 10 January 2014 \\ Number of times this article has been viewed
}

\author{
Harsha Shanthanna' \\ Philip Chan' \\ James McChesney' \\ Lehana Thabane ${ }^{2}$ \\ James Paul' \\ 'Department of Anesthesia, \\ ${ }^{2}$ Department of Clinical \\ Epidemiology and Biostatistics, \\ St Joseph's Hospital, McMaster \\ University, Hamilton, ON, Canada
}

Correspondence: Harsha Shanthanna Department of Anesthesia, St Joseph's Hospital, McMaster University,

50 Charlton Avenue East, Hamilton,

ON, Canada L8N 4A6

Tel + I 905522 II 55 ext 33853

Fax +19055216019

Email harshamd@gmail.com

\begin{abstract}
Background: No proof of efficacy, in the form of a randomized controlled trial (RCT), exists to support pulsed radiofrequency (PRF) treatment of the dorsal root ganglion (DRG) for chronic lumbar radicular (CLR) pain. We determined the feasibility of a larger trial (primary objective), and also explored the efficacy of PRF in decreasing pain on a visual analog scale (VAS) and improving the Oswestry Disability Index.
\end{abstract}

Methods: This was a single-center, placebo-controlled, triple-blinded RCT. Patients were randomized to a placebo group (needle placement) or a treatment group (PRF at $42^{\circ} \mathrm{C}$ for 120 seconds to the DRG). Patients were followed up for 3 months post procedure. Outcomes with regard to pain, Oswestry Disability Index score, and side effects were analyzed on an intentionto-treat basis.

Results: Over 15 months, 350 potential patients were identified and 56 were assessed for eligibility. Fifteen of them did not meet the selection criteria. Of the 41 eligible patients, 32 (78\%) were recruited. One patient opted out before intervention. Three patients were lost to follow-up at 3 months. Mean VAS differences were not significantly different at 4 weeks $(-0.36,95 \%$ confidence interval $[\mathrm{CI}],-2.29,1.57)$ or at 3 months $(-0.76,95 \% \mathrm{CI},-3.14,1.61)$. The difference in mean Oswestry Disability Index score was also not significantly different at 4 weeks $(-2 \%, 95 \% \mathrm{CI},-14 \%, 10 \%)$ or 3 months $(-7 \%, 95 \% \mathrm{CI},-21 \%, 6 \%)$. There were no major side effects. Six of 16 patients in the PRF group and three of 15 in the placebo group showed a $>50 \%$ decrease in VAS score.

Conclusion: The recruitment rate was partially successful. At 3 months, the relative success of PRF-DRG was small. A large-scale trial to establish efficacy is not practically feasible considering the small effect size, which would necessitate recruitment of a challengingly large number of participants over a number of years. Until clear parameters for application of PRF are established, clinicians will need to use their individual judgment regarding its clinical applicability, given the present evidence.

Keywords: pulsed radiofrequency, radicular pain, dorsal root ganglion

\section{Introduction}

Pulsed radiofrequency (PRF) was developed as a modification of the well-known radiofrequency ablation treatment. In conventional radiofrequency ablation, a high frequency alternating current is used to produce coagulative necrosis of the target nerve tissue without any selectivity for nociceptive fibers. ${ }^{1,2}$ However, in PRF, a current in short (20 msec) high voltage bursts is followed by silent phases (480 msec) which allow for heat dissemination, keeping the target tissue controlled below $42^{\circ} \mathrm{C}$. The mechanisms via 
which PRF causes analgesia are still not clearly understood, but laboratory experiments have highlighted some possible ways in which it might act, including its effects on neuropathic pain. ${ }^{3}$ Clinical use of PRF has been expanding, despite there being limited evidence of clinical efficacy in the form of randomized controlled trials (RCTs). Chronic lumbar radicular (CLR) pain is a term used to describe neuropathic pain symptoms in the distribution of a particular lumbar nerve root due to disc protrusion, spinal stenosis, facet hypertrophy, or fibrosis after previous surgery. The pathophysiology of CLR pain involves mechanical, inflammatory, and immunologic factors that affect the function of the dorsal root ganglion (DRG). ${ }^{4}$

Epidural steroid injections are commonly performed but are not effective in all patients and have several limitations to their use. Pulsed radiofrequency of the dorsal root ganglion (PRF-DRG) is a potentially attractive alternative to epidural steroid injection in the treatment of CLR pain. It is target-specific and avoids the use of steroids, thereby eliminating potential side effects such as water retention and endocrine changes like glucose intolerance and adrenal suppression. ${ }^{5}$ Steroids are also known to be associated with potentially serious side effects, such as spinal cord infarction and death secondary to intra-arterial injection of particulate steroid preparations. ${ }^{6}$ There have been few RCTs using PRFDRG for radicular pain. Van Zundert et al performed an RCT in subjects with cervical radicular pain. ${ }^{7}$ Simopoulos et al did a pilot study on lumbar radicular pain, but the methodology included application of conventional radiofrequency over PRF in the study group and was not an efficacy trial. ${ }^{8}$ As such, the efficacy of PRF-DRG in CLR has never been determined.

The objective of this trial was to look at the feasibility of assessing the efficacy of PRF-DRG as compared with placebo needle stimulation in reducing CLR pain. The primary objective was to determine the recruitment rate and completeness of follow-up. Estimation of treatment effects to allow for a formal sample size calculation were considered as secondary outcomes, as they were only exploratory. These were, a decrease in pain score at 4 weeks and up to 3 months after treatment as measured by a visual analog scale (VAS, $0-10$ ), a decrease in Oswestry Disability Index (ODI) at 4 weeks and up to 3 months after treatment, and side effects (both short-term and long-term) up to 3 months. The study protocol has been published previously. ${ }^{9}$

\section{Materials and methods}

This study was approved by the research ethics board at St Joseph's Healthcare Hamilton and is registered at clinicaltrials.gov with the unique identifier NCT01117870. All involved patients gave their written, fully informed consent for the study. This was a single-center, parallelgroup, placebo-controlled, triple-blinded (patients, caregivers, and outcome assessors) RCT. The inclusion criteria were as follows: age 18 years or older; a history of CLR pain of at least 4 months' duration; an average pain score of $\geq 5$ on a VAS of $0-10$; and failure of conservative therapy (eg, physiotherapy, medication trial). The patients were further screened for clinical features of lumbosacral radicular pain. This included segmental pain of a radicular nature originating from the lumbar or sacral segments and radiating below the knee joint, and with a shooting or lancinating quality corresponding to a dermatome suggestive of the involved nerve root. All included patients also had to demonstrate computed tomography/magnetic resonance imaging findings of pathology concordant with the side and level of their clinical features. We did not specify any diagnostic test for inclusion because all of these tests, including the wellknown straight leg raising test, have low diagnostic value. ${ }^{10}$ Exclusion criteria included patient refusal to participate, any contraindication to neuraxial injections, a history of predominant back pain over leg pain, significant anatomic deformity (either congenital or acquired) making it difficult to access the foramen as evidenced by computed tomography/magnetic resonance imaging, severe psychiatric illness, presence of cancer accounting for back pain, inability to communicate in English, allergy to local anesthetics or contrast medium, and a history of motor findings in the affected leg.

Patients with suspected leg pain were initially approached by the research assistant. Further screening for eligibility was done in the presence of the physician. Suitable patients met with the research assistant (blind to intervention), who noted down the baseline parameters of the patient after obtaining an informed consent. All patients were informed that they could withdraw from the study at any time or request rescue analgesia in the form of appropriate analgesics and/or a transforaminal epidural steroid injection.

Allocation to each group was done in a 1:1 ratio, with allocation block sizes of 2, 4, and 6. Enrolled patients were randomized on the day of study intervention at a central location by a single research person who was not involved in any other part of the study. The allocation was given to the assistant in a sealed opaque envelope to be handed over to the nurse operating the radiofrequency machine. All other operating room personnel, including the physician performing the intervention and the patient, were blind to the randomization and treatment. Only the radiofrequency nurse was in view of the working details of the radiofrequency machine, and the noise of the machine was cut off by playing out music. 
After the procedure, the sequence was placed back and sealed, and returned to the central office.

A patient with CLR pain affecting more than one segment on one side was treated for all the involved segments with the same technique and counted as a single intervention for the study. All procedures were performed in the operating room, where patients were positioned prone and procedures were done under fluoroscopy. Lidocaine $1 \%$ was infiltrated at the skin entry site. For both groups, a $10 \mathrm{~cm}, 22$-gauge radiofrequency needle (Baylis Medical, Montreal, QC, Canada) with a $5 \mathrm{~mm}$ curved active tip was used. Target identification and needle positioning were performed similar to the technique described by Simopoulos et al. ${ }^{8} \mathrm{~A}$ radiculogram was done to confirm appropriate placement (Figure 1) and all patients had their respective DRG stimulated for confirmation of the appropriate nerve root involved. Proximity of the needle to the DRG was determined by appropriate sensory stimulation with $50 \mathrm{~Hz}(0.4-0.6 \mathrm{~V})$, and motor stimulation at $2 \mathrm{~Hz}$ was used to determine a threshold 1.5-2.0 times greater than the sensory threshold to avoid placement near the anterior nerve root. The physician instructed the radiofrequency technician to carry out the treatment as randomized, and background music was played throughout the duration of treatment. Study group patients had PRF treatment at $42^{\circ} \mathrm{C}$ for 120 seconds to the DRG. Patients in the control group had low intensity $(<0.2 \mathrm{~V})$ sensory stimulation $(50 \mathrm{~Hz})$, without any active treatment for the same duration. This was done to ensure patient blinding. The assessor (blind to the randomization code) met with all patients in recovery to note any changes and also to inform them that they would be contacted via telephone in the next 24 hours regarding their pain score and side effects. Patients were contacted in person for follow-up at 4 weeks, 2 months, and 3 months post intervention for recording of pain scores, ODI, medication use, and any side effects.
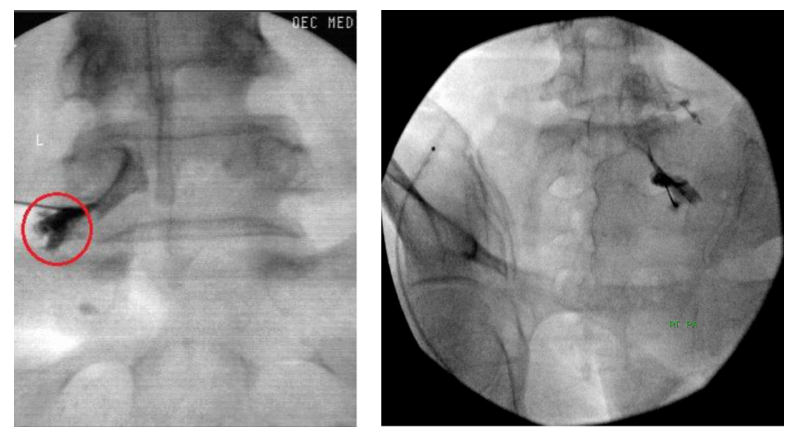

Figure I Radiculogram demonstrating needle placed near the dorsal root ganglion at L5 (left) and SI (right).
We assumed that a sample size of 32 patients (16 in each group) would provide us with sufficiently appreciable differences in clinical efficacy to allow us to calculate the numbers required for a full-scale efficacy study. The study of the efficacy of cervical PRF-DRG showed significant results favoring PRF-DRG for a $20 \%$ pain reduction in VAS score. ${ }^{7}$ Although the investigators planned to enroll 42 patients for the study, they stopped recruitment after accruing 23 patients because these took 2.5 years to recruit. ${ }^{7}$ As an exploratory analysis, we wanted to compare the proportion of patients in each group demonstrating $>50 \%$ relief compared with baseline. It has been noted that a $>30 \%$ reduction in pain appears to reflect at least a moderate clinically important difference, and this needs to be considered in clinical trials. ${ }^{11}$ We aimed at completing the recruitment in 8 months. The expected recruitment of four patients per month was calculated based on transforaminal epidural steroid injections performed over 3-6 months at our center prior to starting the trial. We set a target of recruiting $80 \%$ of patients fulfilling the selection criteria.

\section{Outcome variables and statistical analysis}

Our patient recruitment data are shown in the form of a CONSORT flow diagram (Figure 2). The feasibility outcomes are reported as counts or proportions. The VAS score was calculated on a 10 -point scale between 0 (no pain) and 10 (maximum tolerable pain). The results are reported as the mean and standard deviation and compared using the independent-samples $t$-test. Numbers of patients who had a decrease in pain of $>50 \%$ at 4 weeks were calculated as counts, and analyzed using logistic regression, with the results expressed as the odds ratio (95\% confidence interval) and associated $P$-values. The ODI (version 2.0) was calculated as percent disability, described as the mean \pm standard deviation, and analyzed using the independentsamples $t$-test. The criterion for statistical significance was set at $\alpha=0.05$. Analyses of clinical outcomes were exploratory, so alpha was not adjusted for multiple testing. Side effects were reported as proportions. Any change in medication use was observed as changes in the drug or dose and calculated as number of patients needing a change. All analyses were performed using SAS version 9.2 (SAS Inc, Cary, NC, USA). Because the study was analyzed using the intention-to-treat principle, missing outcomes were imputed using "multiple imputations" for the outcomes of changes in VAS score and ODI, and a 50\% reduction in pain. 


\section{Patient flow through the study from recruitment to analysis}

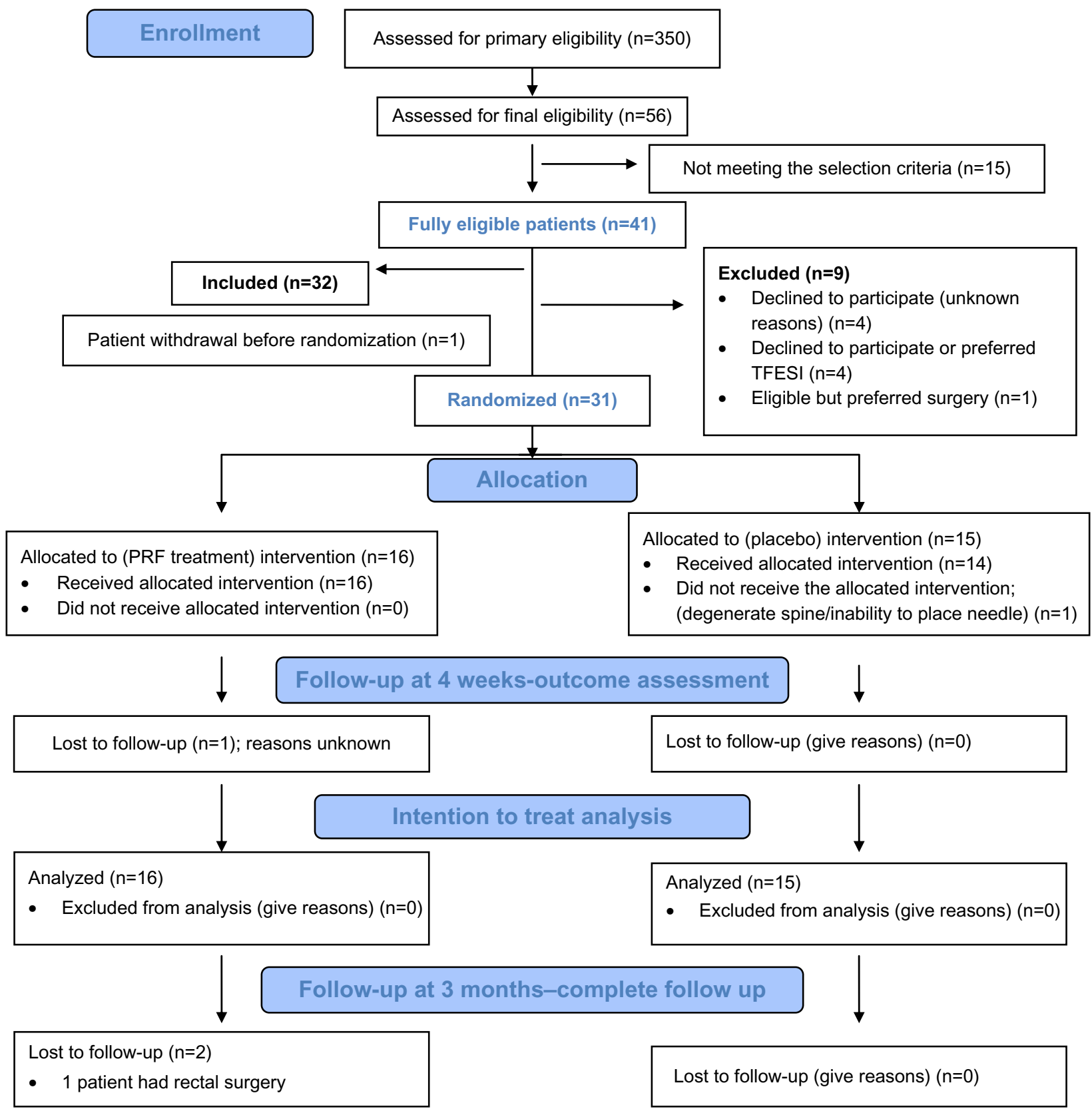

Figure 2 CONSORT flow chart.

Abbreviations: PRF, pulsed radiofrequency; TFESI, transforaminal epidural steroid injection.

\section{Results}

\section{Feasibility outcomes}

For recruitment, 350 patients were screened by the research assistant for medical records indicative of leg pain originating from the back. Only 56 patients were finally assessed for eligibility because the majority of the 350 had nonspecific back pain or inconsistent leg pain. Fifteen patients were further excluded as they did not meet the selection criteria. Of the patients meeting the criteria for inclusion, nine refused to participate. Hence, of 41 fully eligible patients, 32 were recruited over 15 months, resulting in a recruitment rate of $78 \%$. One patient declined to participate after inclusion but before randomization. All patients except one were followed up at 4 weeks $(97 \%)$ and two further patients were lost to follow-up at 3 months. All three of these patients belonged to the PRF group. The rate of loss to follow-up at 3 months was $10 \%$. Patient flow from recruitment to analysis is shown in Figure 2. The demographic variables were comparable 
between the two groups (Table 1). The most likely etiology of CLR pain was determined based on history, examination, and imaging findings (Table 1). All except two patients had one level of treatment. One patient in each group was treated at both the L5 and S1 levels on the same side. Treatment in one patient randomized to the placebo group was abandoned during intervention because we could not place the needle at the DRG.

\section{Clinical efficacy outcomes}

These were considered as exploratory, and the decrease in VAS scores at 4 weeks was considered to be the most important clinical efficacy outcome. All 31 patients who were randomized were included in the statistical analysis. Missing outcomes were imputed using a "multiple imputations" model for clinical outcomes. Comparisons of mean VAS score and ODI are shown as line plots in Figures 3 and 4. The maximum difference in VAS score was observed at 24 hours, with a smaller difference at 4 weeks. VAS scores were again observed to be better in the PRF group at 2 and 3 months. However, none of the differences were statistically significant (Table 2).

ODI scores reflect functional improvement. They were not captured soon after the procedure or at 24 hours, because we did not expect the ODI to change within that short period. The difference in ODI score was larger at 2 and 3 months than at 4 weeks (Table 3). At 4 weeks, five of 16 patients in the PRF group and three of 15 in the placebo group experienced a $>50 \%$ decrease in VAS scores, with an odds ratio of 1.81 (95\% CI 0.36-9.09, $P=0.467)$. There were no major adverse effects of any nature in either group. Two patients in each group complained of minor side effects, such as

Table I Comparison between the two groups for demographic variables, levels of DRG treated, and possible etiology of CLR pain

\begin{tabular}{lll}
\hline & PRF (n=16) & Placebo (n= I 5) \\
\hline $\begin{array}{l}\text { Age (years), median } \\
\text { (minimum, maximum) }\end{array}$ & $62(45-85)$ & $57(35-83)$ \\
$\begin{array}{l}\text { Sex (M, F) } \\
\text { Levels of DRG treated }\end{array}$ & 10,6 & 8,7 \\
L3 & 1 & \\
L4 & 4 & 0 \\
L5 & 10 & 3 \\
SI & 2 & 10 \\
Etiology of CLR pain & & $2(1$ abandoned) \\
Disc-related & 7 & 8 \\
Spinal/foraminal stenosis & 5 & 5 \\
Previous back surgery with fibrosis & 4 & 2 \\
\hline
\end{tabular}

Note: "One patient in each group had both L5 and SI treated on the same side. Abbreviations: PRF, pulsed radiofrequency; CLR, chronic lumbar radicular; DRG, dorsal root ganglion. headache and a transient increase in back pain, which did not last beyond one day. Two patients in the PRF group and one in the placebo group discontinued their use of anti-inflammatory analgesic medication.

\section{Discussion}

Our feasibility was partially successful with regard to the expected recruitment rate, ie, $78 \%$ of the finally eligible patients compared with $80 \%$. However, this took 15 months, with a loss to follow-up of nearly $10 \%$ at 3 months. Being exploratory, the study was not sufficiently powered to detect the efficacy of PRF-DRG over placebo in CLR pain. At 4 weeks and 3 months, the mean VAS score showed a small but nonsignificant decrease in the PRF group compared with the placebo group. Compared with placebo, the decrease in pain scores was consistent throughout follow-up and was more pronounced at 3 months than at 4 weeks. The ODI also decreased in both groups. Similar to VAS scores, the decrease in ODI was more obvious in the PRF group than in the placebo group. In total, five of $16(31 \%)$ patients had a $>50 \%$ decrease in pain score in the PRF group compared with three of $14(20 \%)$ in the placebo group. None of the above outcomes were statistically significant. No side effects or major changes in analgesic medications were observed.

Although evidence-based medicine demands rigorously conducted proof-of-concept trials to demonstrate clinical efficacy, many present-day interventions in practice have not been bench-tested. ${ }^{12}$ The widespread use of PRF for a variety of chronic pain conditions, without the necessary scientific evidence, has been questioned by several authors. ${ }^{2,13,14}$ Observational trials always have a higher chance of overestimating treatment effects and are considered inferior to RCTs in terms of hierarchy of evidence. If they could provide large and consistent treatment effects, they could be rated higher. ${ }^{15}$

Several observational studies have investigated the effects of PRF-DRG on radicular pain. Abejon et al reported use of PRF-DRG in 54 patients with CLR pain. ${ }^{16}$ At 60 days, they observed a significant decrease in pain scores in all patients with herniated disc and spinal stenosis, but not in patients with failed back surgery syndrome. Similar success rates were reported by Teixeira et al in eleven of 13 patients, with relief lasting 11-23 months. ${ }^{17}$ Tsou et al reported a large case series of 127 patients, of whom 49 had predominantly CLR pain; 27 of $49(55 \%)$ patients at 3 months and 20 of 45 (44\%) at one year experienced a $>50 \%$ decrease in pain scores. ${ }^{18}$ Chao et al also reported a large case series of 116 patients with CLR pain; 52 of 116 (44\%) experienced pain relief 


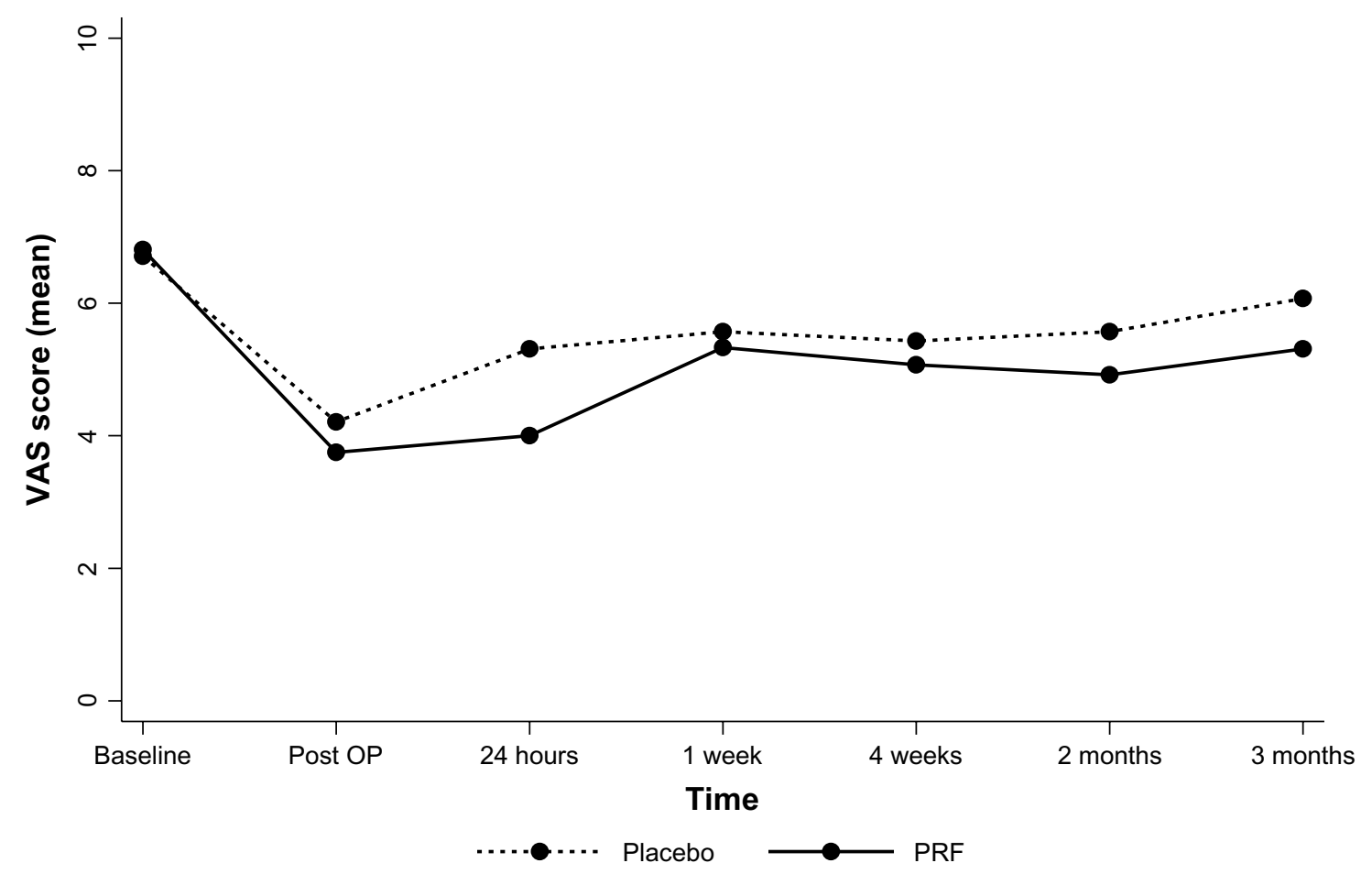

Figure 3 Comparison of mean visual analog scores between the two groups shown as a plot over time. Abbreviations: OP, operation; PRF, pulsed radiofrequency; VAS, visual analog scale.

of $>50 \%$ at 3 months. Interestingly, they had 22 patients with failed back surgery syndrome, and no relative failures compared with patients suffering from herniated discs. ${ }^{19}$ A recent audit by Van Boxem et al reported a success rate of $29 \%$ in 60 patients with CLR pain. ${ }^{20}$
The success rates of the above case series suggest that PRF-DRG might be effective in only $30 \%-50 \%$ of patients with CLR pain. The RCT by Simopoulos et al involved 76 patients randomized to PRF or PRF followed by continuous radiofrequency at the DRG. ${ }^{8}$ At 8 weeks, both groups

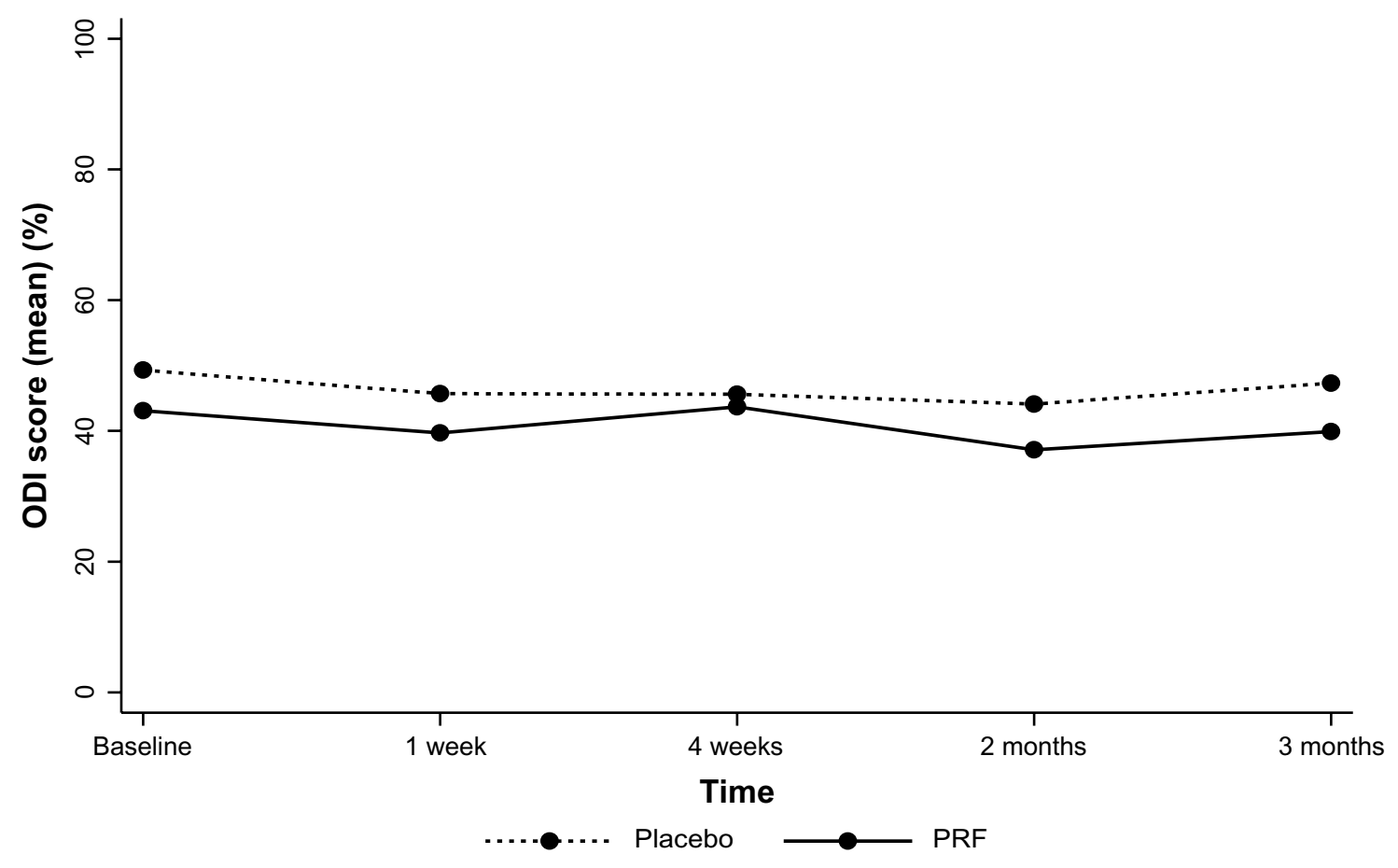

Figure 4 Comparison of mean Oswestry Disability Index (ODI) scores between the two groups shown as a plot over time. Abbreviation: PRF, pulsed radiofrequency. 
Table 2 Differences between the two groups in VAS score post intervention

\begin{tabular}{lll}
\hline Time point & Difference (PRF - placebo) & P-value \\
\hline 24 hours & $-1.68(-0.43,0.96)$ & 0.198 \\
I week & $-0.37(-3.82,3.08)$ & 0.817 \\
4 weeks & $-0.61(-2.68,1.46)$ & 0.552 \\
2 months & $-1.15(-3.61,1.31)$ & 0.342 \\
3 months & $-0.75(-3.12,1.63)$ & 0.524 \\
\hline
\end{tabular}

Notes: Data are shown as the mean and $95 \%$ confidence interval; the independentsamples $t$-test was used to test for statistical significance.

Abbreviations: VAS, visual analog scale; PRF, pulsed radiofrequency.

showed a decrease in pain scores of $>50 \%$, so addition of continuous radiofrequency did not seem to provide any added benefit. Using a similar methodology, Nagda et al reported a retrospective chart review of 40 patients with CLR pain who had shown initial success with PRF and continuous radiofrequency treatment. ${ }^{21}$ Their observation of these patients suggests that repeat treatments of "combined PRF and CRF-DRG" provide pain relief lasting 4.2-4.5 months on average in responsive patients. However, it is not possible to ascribe clinical improvement to either PRF or continuous radiofrequency individually in both of the above studies.

In their review of the use of PRF in chronic pain, Chua et al could identify only six RCTs,${ }^{22}$ of which only one ${ }^{8}$ was done in lumbar radicular pain. Until now, there have only been two efficacy trials for PRF as compared with sham treatment. Van Zundert et al performed a sham-controlled RCT in patients with cervical radicular pain. ${ }^{7}$ Despite stopping enrollment after 23 patients instead of the estimated 42, a decrease in VAS $(0-100)$ of $>20$ points was achieved in nine of eleven patients (82\%) in the PRF group and three of $12(25 \%)$ in the sham group. There are several successful case reports or case series of peripheral PRF used for sacroiliac joint pain, ${ }^{23}$ shoulder pain, inguinal pain, thoracic pain, knee joint pain, occipital neuralgia, and painful neuroma. ${ }^{3}$ Disappointingly, the only published sham-controlled RCT for peripheral neuropathic pain, reported by Akural et al, failed to demonstrate clinical efficacy. ${ }^{24}$ They could not

Table 3 Difference between the two groups for ODI* scores post intervention

\begin{tabular}{lll}
\hline Time point & Difference (PRF - placebo) & P-value \\
\hline I week & $-0.06(-0.24,0.12)$ & 0.485 \\
4 weeks & $-0.03(-0.17,0.11)$ & 0.658 \\
2 months & $-0.08(-0.22,0.06)$ & 0.254 \\
3 months & $-0.08(-0.22,0.07)$ & 0.278 \\
\hline
\end{tabular}

Notes: Data are shown as the mean and $95 \%$ confidence interval; the independentsamples $t$-test was used to test for statistical significance; $* O D I$ scores to be multiplied by 100 for percentage disability.

Abbreviations: ODI, Oswestry Disability Index; PRF, pulsed radiofrequency. perform a sample size calculation, and of 45 well selected patients with limb symptoms, only seven (including four sham-treated patients) achieved a $>30 \%$ decrease in pain score. Similarly, an RCT for trigeminal neuralgia involving 40 patients reported that only two of 20 patients had successful pain relief with $\mathrm{PRF}^{25}$ despite there being previous successful case reports of trigeminal PRF. ${ }^{26}$

The safety of PRF relative to that of continuous radiofrequency is acceptable, with very few reports of significant adverse effects. As observed by most other investigators, we observed only minor side effects, such as headache or procedure-related pain.

RCTs in interventional pain medicine pose unique challenges and dilemmas which have been highlighted before. ${ }^{12,27}$ Of the 350 patients deemed to have CLR pain, only 41 were found to be eligible for our study. Rigorous selection of patients is a major consideration, and a majority of our patients with suspected leg pain had other confounding issues, such as back pain and an inconsistent pattern of leg pain. Our pilot efficacy trial for the use of PRF-DRG in CLR pain demonstrates that the clinical difference, calculated as a $>50 \%$ pain reduction, is quite small, so a large-scale study to demonstrate a statistically significant difference would involve large patient numbers. The trial was initially planned as a comparison between PRF-DRG and transforaminal epidural steroid injection; however, the need to demonstrate efficacy against a sham intervention was considered appropriate to estimate the clinical effect size. In pain trials, clinical improvement can be demonstrated as a decrease in mean pain scores between groups, ie, as a continuous outcome measure, or as a binary outcome measure in the form of the proportion of individuals who demonstrate a successful response. Although a continuous outcome measure could require a smaller sample size, comparing mean pain scores could be inappropriate because group data may mask good responses occurring in subgroups or in certain patients. ${ }^{28}$ Therefore, we feel it is appropriate to consider the number of successful patients in each group. Sample size calculation with imputation of missed outcomes for VAS scores at 4 weeks $(>50 \%$ pain relief) suggests that we would need 253 patients in each group, given the present result for PRF (5/16 versus $3 / 15$ for placebo). Although other trials have investigated $>20 \%$ pain relief, ${ }^{7}$ Dworkin et al and Yelland et al have shown that this is not worthwhile for patients. ${ }^{11,29}$

Our study could be criticized for including patients with different pathologies contributing to CLR pain. However, larger case series have not shown a difference in response rates to treatment between disc-related and other groups. ${ }^{19}$ In practice, 
most patients have a mixture of degenerative spinal disease involving spondylolisthesis, disc changes, facet arthropathy, and foraminal or lateral recess stenosis. More specific selection of patients would limit recruitment opportunities further. Another limitation of the study could be that we did not include a diagnostic root block to select the appropriate level. However, this limitation was successfully overcome by stimulating (testing) the respective DRG to confirm the appropriate level of clinical symptoms and imaging findings. ${ }^{30}$

Despite being used in clinical practice for more than a decade, the optimal parameters for application of PRF have yet to be determined. According to Cosman and Cosman, ${ }^{31}$ unlike continuous radiofrequency, for which we have existing guidelines, achieving optimal parameters for a desired clinical objective using PRF will need more experience and a better understanding of the electrical and thermal field effects on neurons. They suggest that the endpoint effect on neurons could be an electrical dose function at the position of the neuron and the electrical dose is likely to be a graded function of exposure time. There is wide variation in the literature on the reported duration of PRF. Although most studies have applied PRF for 120 seconds, others claim success with application for 4-10 minutes ${ }^{32,33}$ and some have also used a steroid at the end of treatment, which is an active treatment in itself. ${ }^{34}$

\section{Conclusion}

Our results suggest that clinical improvements with PRFDRG as reflected in the VAS score are small in patients with CLR pain. Our results suggest that a large-scale clinical trial is not feasible because this would require a large sample size and could take a number of years to recruit. Options to consider may include modifications to the selection criteria and use of a multicenter study design. Although a lesser degree of pain reduction $(<20 \%)$ could be considered, it would not be clinically appropriate. Many basic science experiments have shown that PRF triggers a genuine neurobiologic phenomenon, and its analgesic effect may involve more than one specific mechanism. Its clinical effect might also involve different thresholds for different clinical targets. Until we establish correct parameters for application of PRF, the appropriate patient population, and/or the pain conditions that are most responsive to PRF, we may have to use our individual judgment regarding its clinical applicability, given the present evidence.

\section{Acknowledgment}

This trial received funding from the Canadian Pain Society in the form of a Trainee Research Award, Clinical Science
Category, 2011, awarded to the main author (HS) who was working as a clinical fellow in the initial phase of this trial.

\section{Disclosure}

The authors report no conflicts of interest in this work.

\section{References}

1. Byrd D, Mackey S. Pulsed radiofrequency for chronic pain. Curr Pain Headache Rep. 2008;12:37-41.

2. Bogduk N. Pulsed radiofrequency. Pain Med. 2006;7:396-407.

3. Cahana A, Van Zundert J, Macrea L, van Kleef M, Sluijter M. Pulsed radiofrequency: current clinical and biological literature available. Pain Med. 2006;7:411-423.

4. Howe JF, Loeser JD, Calvin WH. Mechanosensitivity of dorsal root ganglia and chronically injured axons: a physiological basis for the radicular pain of nerve root compression. Pain. 1977;3:25-41.

5. Weinstein SM, Herring SA; NASS. Lumbar epidural steroid injections. Spine J. 2003;3(Suppl 3):37S-44S.

6. Benedetti E, Siriwetchadarak R, Stanec J, Rosenquist R. Epidural steroid injections: complications and management. Tech Reg Anesth Pain Manag. 2009;13:236-250.

7. Van Zundert J, Patijn J, Kessels A, Lamé I, van Suijlekom H, van Kleef M. Pulsed radiofrequency adjacent to the cervical dorsal root ganglion in chronic cervical radicular pain: a double blind sham controlled randomized clinical trial. Pain. 2007;127:173-182.

8. Simopoulos TT, Kraemer J, Nagda JV, Aner M, Bajwa ZH. Response to pulsed and continuous radiofrequency lesioning of the dorsal root ganglion and segmental nerves in patients with chronic lumbar radicular pain. Pain Physician. 2008;11:137-144.

9. Shanthanna H, Chan P, McChesney J, Paul J, Thabane L. Assessing the effectiveness of 'pulse radiofrequency treatment of dorsal root ganglion' in patients with chronic lumbar radicular pain: study protocol for a randomized control trial. Trials. 2012;13:52.

10. Iversen T, Solberg TK, Romner B, et al. Accuracy of physical examination for chronic lumbar radiculopathy. BMC Musculoskelet Disord. 2013;14:206.

11. Dworkin RH, Turk DC, Wyrwich KW, et al. Interpreting the clinical importance of treatment outcomes in chronic pain clinical trials: IMMPACT recommendations. J Pain. 2008;9:105-121.

12. Bogduk N, Fraifeld EM. Proof or consequences: who shall pay for the evidence in pain medicine? Pain Med. 2010;11:1-2.

13. Kvarstein G. Pulsed radiofrequency - time for a clinical pause and more science. Scandinavian Journal of Pain. 2012;3:124-126.

14. Cohen SP, Van Zundert J. Pulsed radiofrequency: rebel without cause. Reg Anesth Pain Med. 2010;35:8-10.

15. Guyatt GH, Oxman AD, Sultan S, et al. GRADE guidelines: 9. Rating up the quality of evidence. J Clin Epidemiol. 2011;64:1311-1316.

16. Abejón D, Garcia-del-Valle S, Fuentes ML, Gómez-Arnau JI, Reig E, van Zundert J. Pulsed radiofrequency in lumbar radicular pain: clinical effects in various etiological groups. Pain Pract. 2007;7:21-26.

17. Teixeira A, Grandinson M, Sluijter ME. Pulsed radiofrequency for radicular pain due to a herniated intervertebral disc - an initial report. Pain Pract. 2005;5:111-115.

18. Tsou HK, Chao SC, Wang CJ, et al. Percutaneous pulsed radiofrequency applied to the L-2 dorsal root ganglion for treatment of chronic low-back pain: 3-year experience. J Neurosurg Spine. 2010;12: 190-196.

19. Chao SC, Lee HT, Kao TH, et al. Percutaneous pulsed radiofrequency in the treatment of cervical and lumbar radicular pain. Surg Neurol. 2008;70:59-65.

20. Van Boxem K, van Bilsen J, de Meij N, et al. Pulsed radiofrequency treatment adjacent to the lumbar dorsal root ganglion for the management of lumbosacral radicular syndrome: a clinical audit. Pain Med. 2011;12:1322-1330. 
21. Nagda JV, Davis CW, Bajwa ZH, Simopoulos TT. Retrospective review of the efficacy and safety of repeated pulsed and continuous radiofrequency lesioning of the dorsal root ganglion/segmental nerve for lumbar radicular pain. Pain Physician. 2011;14:371-376.

22. Chua NH, Vissers KC, Sluijter ME. Pulsed radiofrequency treatment in interventional pain management: mechanisms and potential indications - a review. Acta Neurochir (Wien). 2011;153:763-771.

23. Vallejo R, Benyamin RM, Kramer J, Stanton G, Joseph NJ. Pulsed radiofrequency denervation for the treatment of sacroiliac joint syndrome. Pain Med. 2006;7:429-434.

24. Akural E, Järvimäki V, Korhonen R, Kautiainen H, Haanpää M. Pulsed radiofrequency in peripheral posttraumatic neuropathic pain: a double blind sham controlled randomized clinical trial. Scandinavian Journal of Pain. 3:127-131.

25. Erdine S, Ozyalcin NS, Cimen A, Celik M, Talu GK, Disci R. Comparison of pulsed radiofrequency with conventional radiofrequency in the treatment of idiopathic trigeminal neuralgia. Eur J Pain. 2007;11:309-313.

26. Van Zundert J, Brabant S, Van de Kelft E, Vercruyssen A, Van Buyten JP. Pulsed radiofrequency treatment of the Gasserian ganglion in patients with idiopathic trigeminal neuralgia. Pain. 2003;104:449-452.

27. Van Zundert J, Van Boxem K, Joosten EA, Kessels A. Clinical trials in interventional pain management: optimizing chances for success? Pain. 2010;151:571-574.
28. Ghahreman A, Ferch R, Bogduk N. The efficacy of transforaminal injection of steroids for the treatment of lumbar radicular pain. Pain Med. 2010;11:1149-1168.

29. Yelland MJ, Schluter PJ. Defining worthwhile and desired responses to treatment of chronic low back pain. Pain Med. 2006;7:38-45.

30. Wolff AP, Groen GJ, Crul BJ. Diagnostic lumbosacral segmental nerve blocks with local anesthetics: a prospective double-blind study on the variability and interpretation of segmental effects. Reg Anesth Pain Med. 2001;26:147-155.

31. Cosman ER Jr, Cosman ER Sr. Electric and thermal field effects in tissue around radiofrequency electrodes. Pain Med. 2005;6: $405-424$.

32. Akkoc Y, Uyar M, Oncu J, Ozcan Z, Durmaz B. Complex regional pain syndrome in a patient with spinal cord injury: management with pulsed radiofrequency lumbar sympatholysis. Spinal Cord. 2008;46: 82-84.

33. Navani A, Mahajan G, Kreis P, Fishman SM. A case of pulsed radiofrequency lesioning for occipital neuralgia. Pain Med. 2006;7: 453-456.

34. Philip CN, Candido KD, Joseph NJ, Crystal GJ. Successful treatment of meralgia paresthetica with pulsed radiofrequency of the lateral femoral cutaneous nerve. Pain Physician. 2009;12:881-885.
Journal of Pain Research

\section{Publish your work in this journal}

The Journal of Pain Research is an international, peer-reviewed, open access, online journal that welcomes laboratory and clinical findings in the fields of pain research and the prevention and management of pain. Original research, reviews, symposium reports, hypothesis formation and commentaries are all considered for publication.

\section{Dovepress}

The manuscript management system is completely online and includes a very quick and fair peer-review system, which is all easy to use. Visit http://www.dovepress.com/testimonials.php to read real quotes from published authors. 